\title{
Integrating autonomous aerial scouting with autonomous ground actuation to reduce chemical pollution on crop soil
}

\author{
Jesús Conesa-Muñoz ${ }^{1}$, João Valente ${ }^{2,3}$, Jaime del Cerro ${ }^{2}$, Antonio Barrientos ${ }^{2}$, Ángela \\ Ribeiro $^{1 *}$ \\ ${ }^{1}$ Centre for Automation and Robotics, CSIC-UPM. Arganda del Rey, 28500, Madrid, Spain. \\ \{jesus.conesa, angela.ribeiro\}@csic.es \\ ${ }^{2}$ Centre for Automation and Robotics, UPM-CSIC, José Gutierrez Abascal 2, 28006, Madrid, \\ Spain \\ \{joao.valente, j.cerro, antonio.barrientos\}@upm.es \\ Current address: ${ }^{3}$ Carlos III University, Av. Universidad 30, 28911, Leganés, Spain \\ jvalenteding.uc3m.es
}

\begin{abstract}
Many environmental problems cover large areas, often in rough terrain constrained by natural obstacles, which makes intervention difficult. New technologies, such as unmanned aerial units, may help to address this issue. Due to their suitability to access and easily cover large areas, unmanned aerial units may be used to inspect the terrain and make a first assessment of the affected areas; however, these platforms do not currently have the capability to implement intervention.

This paper proposes integrating autonomous aerial inspection with ground intervention to address environmental problems. Aerial units may be used to easily obtain relevant data about the environment, and ground units may use this information to perform the intervention more efficiently.

Furthermore, an overall system to manage these combined missions, composed of aerial inspections and ground interventions performed by autonomous robots, is proposed and implemented.

The approach was tested on an agricultural scenario, in which the weeds in a crop had to be killed by spraying herbicide on them. The scenario was addressed using a real mixed fleet composed of drones and tractors. The drones were used to inspect the field and to detect weeds and to provide the tractors the exact coordinates to only spray the weeds. This aerial and ground mission collaboration may save a large amount of herbicide and hence significantly reduce the environmental pollution and the treatment cost, considering the results of several research works that conclude that actual extensive crops are affected by less than a $40 \%$ of weed in the worst cases
\end{abstract}

Keywords: collaborative inspection and intervention mission, aerial and ground fleet, autonomous fleet, site-specific weed treatment, precision agriculture

adfa, p. 1, 2011.

(C) Springer-Verlag Berlin Heidelberg 2011 


\section{$1 \quad$ Introduction}

Many environmental problems require surveillance or scouting stages previous to the intervention phase that alleviates or solves the problem. Many cases require coverage of large areas, often in rough terrain constrained by natural obstacles, which makes continuous inspections difficult. New technologies, such as unmanned aerial units, may help in this issue due to their suitability to access and easily cover large surfaces. Thus, environmental actuation can be split into two stages: aerial inspection with drones and ground intervention with typically more powerful platforms. The aerial inspection may provide a quick and easy assessment of the affected areas to be used for ground intervention to implement the work more efficiently. The proper integration of aerial and ground units would make the use of the current autonomous robots more efficient for treating environmental disasters, such as oil spills [1], forest fires [2] or earthquakes [3]. Such integration could be applied even in agriculture, where some agricultural tasks, such as weed treatment, might be accomplished by ground units only in the affected zones by following a weed distribution map obtained from the information provided by the aerial units. This site-specific weed management has clear environmental benefices, mainly in extensive crops where research work reported weed infestations around the $40 \%$ in the worst cases [4,5]. In other words, more than a $60 \%$ of herbicide could be potentially saved with the proper technology.

In many contexts, inspection and actuation would be greatly enhanced if performed by autonomous robots and, in particular, for large areas, with fleets of autonomous robots. Moreover, the entire work to be accomplished by the fleet would be more efficient if the autonomy of the whole system was complete, i.e., the fleet of aerial and ground autonomous robots works together without human intervention, which would only be in charge of supervising the work of the fleet. In the following sections, a system designed and developed to accurately treat weeds in field crops with herbicides is described.

In the agricultural context, herbicide application is an important economic and environmental issue. Herbicides are chemical products used to control unwanted plants (weeds) interfering with crops. EU countries used approximately 135,000 tonnes of herbicides in 2007 [6]. These products make a significant contribution to maintaining food production; according to [7], each euro $(€)$ invested in herbicides (and pesticides in general) returns 4 euros in crops saved. Considering that the total sales of herbicides in Europe is currently approximately 3,390 million $€$ per year [8], we can estimate that, in Europe, pesticides may provide over 13,500 million $€$ per year in saved crops. However, such assessments do not consider the indirect, but substantial, environmental and economic costs associated with herbicide use. For example, it has been estimated that only $5 \%$ of herbicides reach the target weeds [9], whereas the bulk of each application (over 95\%) is left to impact the surrounding environment. The economic value of pesticide environmental impact has been estimated to total approximately 8,000 million $\$$ per year in the USA [7], and approximately $50 \%$ of pesticide usage consists of herbicide treatments.

To mitigate the abusive use of herbicides and the consequent chemical pollution on crop soils, precision agriculture was developed as a more environmentally careful 
way to manage fields. Precision agriculture is the application of technologies and principles to manage the spatial and temporal variability associated with all aspects of agricultural production for the purpose of improving crop performance and environmental quality [10]. In this context, aerial inspection missions may be used to easily acquire the variability in fields (that is, the distributions of the crop, weeds, insects, humidity, and soil fertility), and farmers may use these data to work selectively on the fields (also known as site-specific treatments), significantly decreasing the use of agrochemical products (herbicides, insecticides and fertilizers), which are highly dangerous for the environment. There are several studies devoted to crop inspection by analyzing and processing aerial images, for example, to detect weeds [11], and there are works devoted to developing tools [12] and site-specific treatments based on previously acquired knowledge [13,14]. Nevertheless, only the RHEA project [15], in which this work is framed, has linked the two steps to completely automate the sitespecific herbicide treatments. To achieve this goal, this paper uses an autonomous and heterogeneous fleet to implement the entire process autonomously and accurately. The inspection step is accomplished by an aerial team composed of 2 drones and a treatment step using a ground team composed of 3 medium autonomous tractors.

The use of a collaborative heterogeneous fleet for selective treatments is a novel approach that presents several advantages. The benefits of this solution over the conventional large vehicles equipped with many different actuators and sensors arise from different facts summarized in Table 1.

Table 1. Advantages of using a fleet of small/medium sized robots over one large agricultural vehicle

\begin{tabular}{|l|l|l|}
\hline & $\begin{array}{l}\text { Traditional big ma- } \\
\text { chine }\end{array}$ & A fleet of small/medium robots \\
\hline $\begin{array}{l}\text { Safety in auton- } \\
\text { omous operation } \\
\text { mode }\end{array}$ & $\begin{array}{l}\text { Becomes a safety } \\
\text { problem in case of } \\
\text { failure }\end{array}$ & $\begin{array}{l}\text { Small/medium sized robots can interact } \\
\text { with humans in a safer way }\end{array}$ \\
\hline $\begin{array}{l}\text { Fault impact on } \\
\text { mission comple- } \\
\text { tion }\end{array}$ & $\begin{array}{l}\text { A failure will stop the } \\
\text { entire mission until } \\
\text { the machine is re- } \\
\text { paired }\end{array}$ & $\begin{array}{l}\text { Robot teams allow for mission re- } \\
\text { planning in case of failure of one vehi- } \\
\text { cle }\end{array}$ \\
\hline $\begin{array}{l}\text { Impact on the } \\
\text { field }\end{array}$ & $\begin{array}{l}\text { High damage by soil } \\
\text { compaction }\end{array}$ & $\begin{array}{l}\text { Lower compaction (lighter vehicles) } \\
\text { and more precision movements (farm- } \\
\text { ing at plant level) }\end{array}$ \\
\hline Personnel & $\begin{array}{l}\text { An operator for each } \\
\text { vehicle }\end{array}$ & $\begin{array}{l}\text { An operator can supervise several ve- } \\
\text { hicles }\end{array}$ \\
\hline
\end{tabular}

In the following sections, the architecture of the overall system (Mission Manager) designed and developed to integrate aerial scouting missions with ground treatment missions is explained. The employed robot platforms used to implement site-specific weed treatments are described. Finally, the results section explains how the overall system, fleet and implemented Mission Manager, performed an accurate selective treatment in a real crop in an autonomous way. 


\section{Mission Manager Architecture}

In general, even if the robotic platforms used are autonomous, software is required to manage the entire process, that is, an overall system to generate the directions for the units to follow to accomplish their missions, to send them to the platforms, to coordinate the fleet (the vehicles may interfere with each other), to supervise the fleet while working, to report failures to the operator in charge of the fleet, and to process the data acquired by the inspection missions.

Fig. 1 shows the generic architecture that the proposed system, called hereinafter Mission Manager, should have.

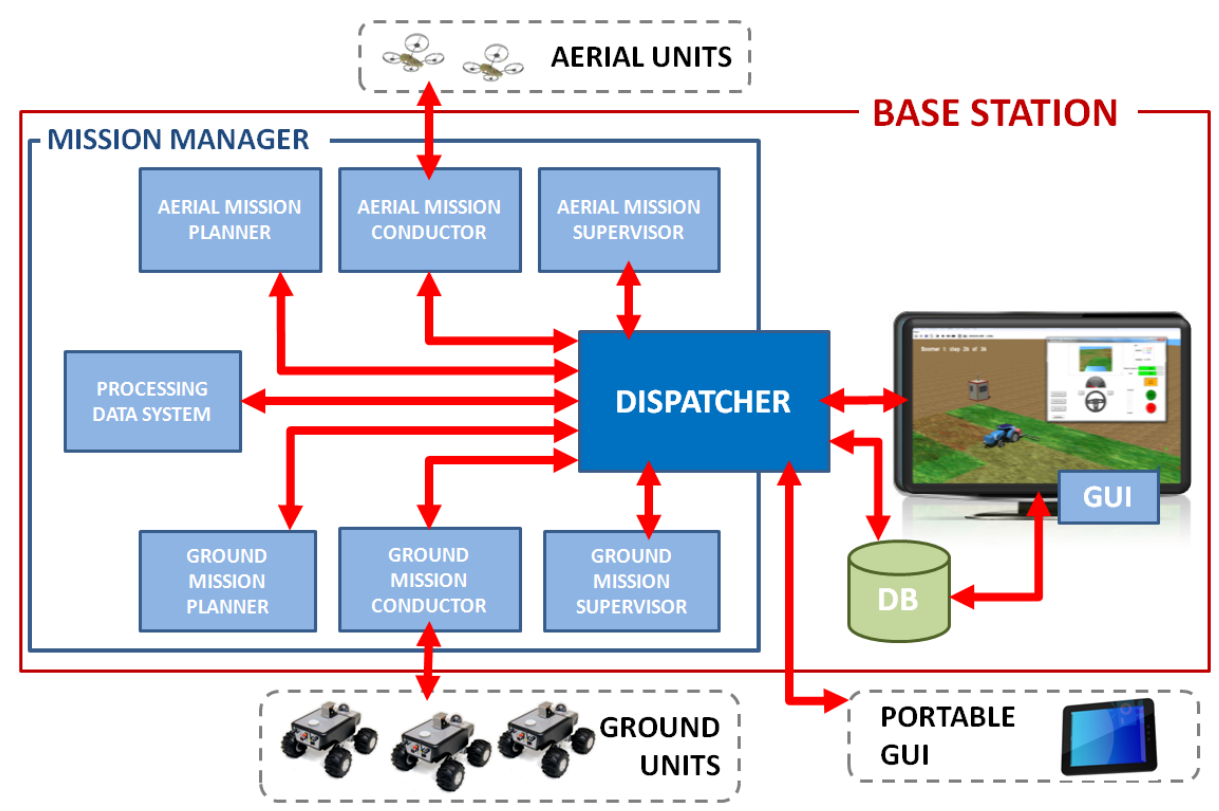

Fig. 1. Architecture of the Mission Manager and its connections with external elements/systems

Thus, the generic Mission Manager is composed of the following modules:

- An aerial and a ground mission planner: To generate the plans that the units have to follow to complete the missions. They cannot be unified in a single planner because of the inherent differences of the aerial and ground units, as well as the different characteristics of their missions (surveillance/scouting and intervention/treatment).

- An aerial and a ground conductor: To automate the mission at the fleet level. Although the units, considered separately, may be autonomous, the fleet has to be coordinated, for instance, to launch/pause/resume/stop the mission for all members simultaneously. Additionally, these conductors are in charge of decoding the calculated plans (and transmitting them) to the exact commands supported by the units. 
- An aerial and a ground mission supervisor: To monitor and corroborate that the missions are executed according to the generated plans. Because the units work in an uncontrolled environment, subject to unpredictable conditions (wind, light, terrain roughness, animals that may suddenly appear, etc.), there may be differences between the planned mission and the execution, for example, small deviations in the trajectories and speed due to wind or the terrain. Once deviations are detected, the supervisors report to the operator by issuing alarms that may be displayed on a GUI (graphical user interface).

- A processing data system: To receive and analyze the raw data acquired by the scouting mission to extract knowledge to be used in the intervention mission. For instance, in an agricultural mission, this module may consist of a mapping system to process the images taken by the aerial units and to detect and obtain the exact coordinates of the weed patches within the field.

- A dispatcher: To manage the workflow required to complete the entire process. To do this, the dispatcher encapsulates the connections to all the modules included into the Mission Manager and redirects the process to the appropriate modules when required. Moreover, it gathers and processes and redirects the queries (plans, executions, pauses, resumes, and aborts) from the external systems (GUI) if the operator wants to actively control the workflow.

This component is particularly important because it allows the connection of new modules to the Mission Manager in order to support new functionalities.

In addition to the Mission Manager internal modules, there are some external systems that may interact with it.

- GUI (Graphical User Interface): Allows the operator to access the Mission Manager. The GUI also displays all the information generated by the Mission Manager (plans, execution states, alarms, etc.) and guides the operator through the different workflow steps.

- Portable GUI: The Mission Manager is intended to be run on a computer hosted in a base station (a cabin with some antennas and a router to create a Wi-Fi network to access the units) next to the affected area. Thus, for those situations in which a breakdown forces the operator to move to the units, it is useful to have a portable GUI to control a particular unit of the fleet outside the cabin.

- Database: Allows register data about the mission, such as plans or the acquired data, to interrupt and resume the process, or even to process offline when the units are not working (for example, the case of processing images or any other big data acquired during the inspection).

\section{Fleet Robots}

In this section, the available fleet of robots used in the former results section is described. The fleet used is the fleet of the European project RHEA [15]. 


\subsection{Aerial fleet}

The aerial fleet was composed of two six-rotor drones (AR200 model), developed by the AirRobot company [16]. Each one was able to carry a sensor-payload up to $1.5 \mathrm{~kg}$ with a fly autonomy of around approximately 40 minutes. Six-rotor units were used to provide certain safety redundancy in case of failure in one motor.

The drones were equipped with two cameras, visible and near infrared spectrum (two Sigma DP2 Merril models, one of them modified to record NIR images), mounted on a gimbal system (see Fig. 2) to reduce vibrations and to allow the cameras to point down when the drones perform steady flights.

The drones accept plans mainly composed of a list of ordered way-points where the drone has to take a picture, and the drones then autonomously fly to the way points.

Drones are able to provide telemetry information during the flight, including information required for supervision, such as position estimation and battery level. After finishing the mission, the drones return to their home points.

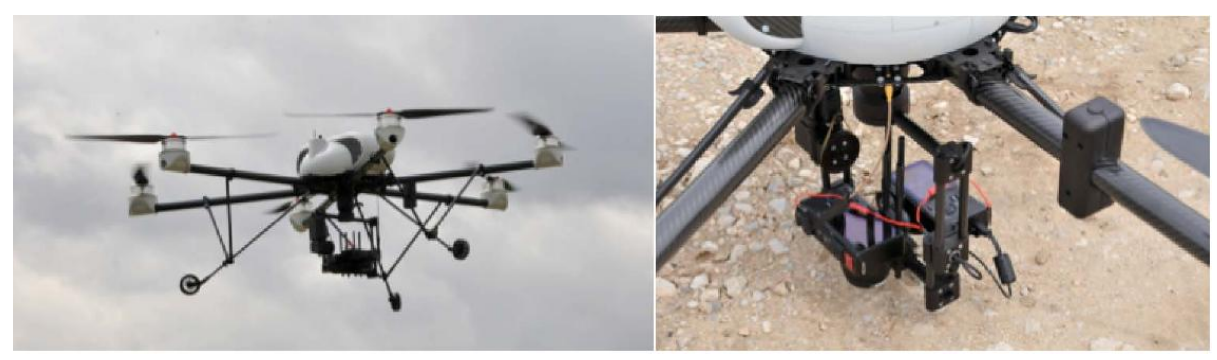

Fig. 2. AR200 drone in flight with a detail of the camera mounting

\subsection{Ground fleet}

The ground fleet was composed of three medium tractors (see Fig. 3), based on a restructured New Holland Boomer $3050(50 \mathrm{hp}, 1270 \mathrm{~kg}$ ) [17], in which the cabin was reduced to mount some of the computer equipment required for the perception, actuation, location, communication and safety systems.

Several sensor systems, such as an RTK-GPS receiver, an RGB camera and a LiDAR, allow autonomous and safe navigation.

The RTK-GPS receiver, a Trimble BX982 model, is a multi-channel, multifrequency OEM GNSS receiver that enables OEM and system integrators to rapidly integrate centimeter-level positioning. The receiver supports two antennas connected in such way that the independent observations from both antennas are passed to the processor, where multi-constellation RTK baselines are computed and compared with the positions provided by both antennas. Because the real physical distance and their positions on the vehicle are known, it is possible to calculate the vehicle's heading with high accuracy. Therefore, a single connection to the tractor receiver (via RS232, USB, Ethernet or CAN) delivers both centimeter-accuracy positions and a heading that is accurate to less than a tenth of a degree ( $2 \mathrm{~m}$ baseline). In this manner, both the 
position and heading of the vehicles are provided with high precision at a maximum frequency of $20 \mathrm{~Hz}$.

The camera onboard each tractor is an SVS4050CFLGEA model from SVSVISTEK (Seefeld, Germany) with a CCD Kodak KAI 04050M/C sensor and a GR Bayer color filter, which provides high-resolution images $(2,336$ by 1,752 pixels with a 5.5 by $5.5 \mu \mathrm{m}$ pixel size) to accurately determine in real time the locations of the weeds, obstacles and crop lines. The camera was placed inside a housing unit with a fan controlled by a thermostat for cooling purposes, which allows it to work even when it is raining or when the temperature is above $50{ }^{\circ} \mathrm{C}$. The description of how the camera detects weed and crop rows (appropriate strategy for wide-row crops, such as maize) is out of the scope of this paper. Actually, the considered scenario only takes into account the weed detection by remote sensing, since it is the proper example to illustrate the integration of the whole elements of the fleet, in other words the scouting mission with the intervention mission.

The LiDAR sensor, an LMS 111 (SICK AG, Waldkirch, Germany), was installed in the middle of the vehicle's front with a push-broom configuration ( $4^{\circ}$ inclination) and was used to detect obstacles along the vehicle trajectory with a ground clearance of $70 \mathrm{~cm}$.

To perform the treatment, the tractor was equipped with a selective sprayer bar developed by Agrosap [12]. This tool is a 6-m spray boom with 12 nozzles, which can be independently activated, and 2 tanks, one to store water $(200 \mathrm{~L})$ and the other, smaller tank to store the herbicide. The sprayer is equipped with a direct injection system that mixes the agrochemical product and water just when a single or several nozzles are opened, which reduces herbicide waste.

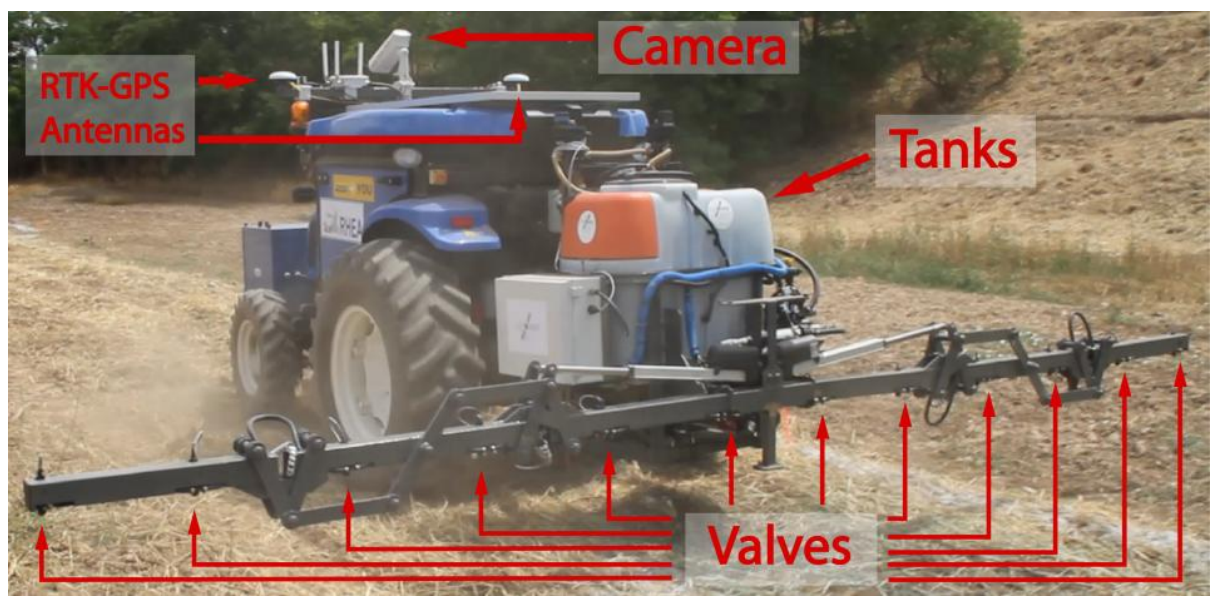

Fig. 3. Ground unit

Finally, the tractors are equipped with an on-board computer, a CompactRIO model 9082 from National Instruments (Austin, TX, USA), which runs the internal control system that manages the sensors and actuators and allows remote control of the unit. Similarly to the aerial units, this internal controller allows autonomous execution of 
some remote commands: move, pause, resume, and stop, including performing a treatment plan. The plan is mainly composed of a list of the way-points the vehicles have to cover and also contains the states for the spraying bar (that is, the nozzles that must be opened and closed) for each point and other mission parameters, such as the speeds. More details about the ground units and their capabilities, such as navigation and control techniques utilized, can be found in [18].

\section{$4 \quad$ Results}

To test the complete set of steps implemented in the Mission Manager, a winter cereal field was prepared containing weed patches. The idea was to autonomously and sequentially execute all the steps required to perform a site-specific herbicide treatment via the Mission Manager running on a computer placed in a cabin situated next to the field and using the aerial and ground units presented in Section 3. The field was located in the experimental CSIC farm "La Poveda" [40 $\left.18^{\prime} 51.102^{\prime \prime} \mathrm{N}, 3^{\circ} 29^{\prime} 03.379^{\prime \prime} \mathrm{W}\right]$ in Arganda del Rey. The field was $2,400 \mathrm{~m}^{2}$ and was treated using a pre-emergency herbicide, except for nine $3 \mathrm{~m}$ x $3 \mathrm{~m}$ square areas (see Fig. 4), where some weeds (Sinapis arvensis) were seeded.

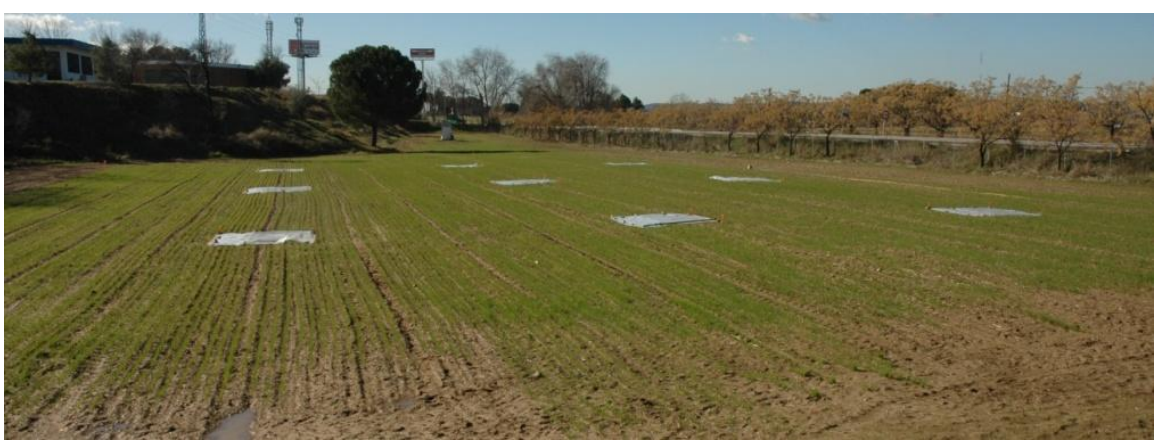

Fig. 4. Winter cereal field prepared to contain nine weed patches

The field contour (yellow rectangle on Fig. 5) was acquired using a GPS and was stored in the database. Via the GUI, an aerial scouting plan was requested. The aerial planner automatically built a safety border (green contour on Fig. 5) expanding some margins of the field contour (this border cannot be exceeded by the drones) and calculated (from the contour of the field, the flight attitude, the resolution and the size of the images provided by the cameras) the way-points where the drones need to take images to sample the entire field. This information is used later to create the weed distribution map. The obtained routes for each drone are the red and blue lines represented in Fig. 5 a.

Once planned, the aerial mission conductor requested the launch of the scouting mission, and the operator in charge of supervision approved the start of the mission. The plans were automatically loaded into the units, and the supervisor pilot was asked (via GUI) to approve take-off (required by the Spanish drone regulation) until the 
initial attitude specified in the plan was reached. Then, the drones executed the inspection following the trajectories shown in Fig. 5b. The aerial supervisor (module that is part of the Mission Manager) monitored the mission, and non-failures were detected during the execution of the aerial mission.

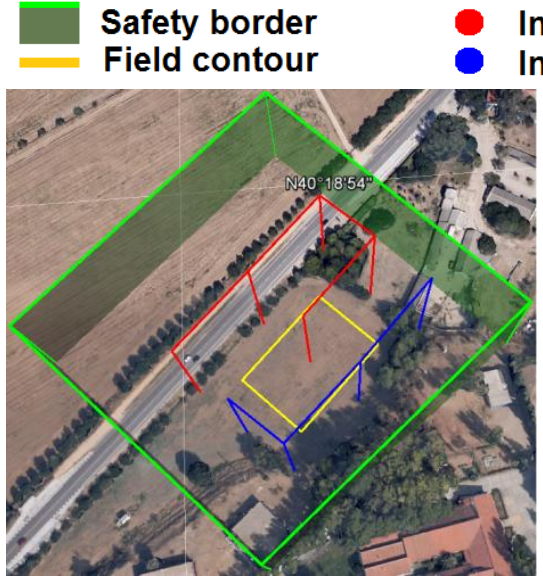

(a)

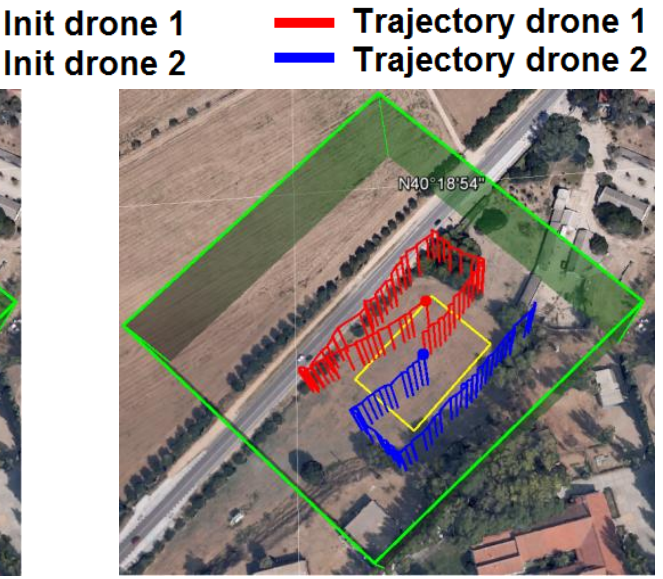

(b)

Fig. 5. Inspection aerial mission: a) planned trajectories and b) real trajectories

Once the drone's plans were completed, the Mission Manager requested a landing maneuver from the supervisor pilot and, once on the ground, the cameras' cards with the images were manually removed and inserted into the computer running the Mission Manager. The processing data system was invoked by the Dispatcher (module that is part of the Mission Manager). The processing data, in this case, consists of a weed detection system composed by a mosaicking system [19] and mapping module [11] developed by the IRSTEA and IAS-CSIC groups, respectively. The system outputs a weed distribution map, which is used by the ground Planner to develop the plan for treatment. Unfortunately, the weeds did not grow as expected and did not have the shape of the expected patches (see Fig. 6a). Consequently, the obtained distribution map, although it contained the real patch shapes, did not have the expected squares, making it difficult to determine whether the herbicide was sprayed on the appropriate areas. For this reason, the expected map was built artificially (Fig. 7) and was used to generate the treatment plan. The trajectories were optimized to reduce fuel, so the planner decided to use only one tractor.

The expected patches were covered with paper (Fig. 6b). A total of five paper strips were used in each patch, arranged in parallel and spaced 1 meter (three strips inside and two outside of the patch), for measuring the on/off time lag and therefore the percentage of the target area sprayed and not sprayed by water mixed with colorant. Then the treatment mission was executed. The ground supervisor (module that is part of the Mission Manager) monitored the mission, and non-failures were detected during the execution of the treatment mission; in fact the real trajectories were nearly the same (deviations of less than $7 \mathrm{~cm}$ ) as the planned trajectories (Fig. 7). Moreover, 
the sprayed surface accurately matched the weeds, in fact the results showed that the spraying operation successfully sprayed more than $97 \%$ of the target area (i.e., weed patches) without any spraying in non-target areas (i.e., weed-free areas).

Only six of the nine patches were covered because the right part of the field was reserved for intermediate tests. The entire test can be played on [20] as part of an RHEA project demo.

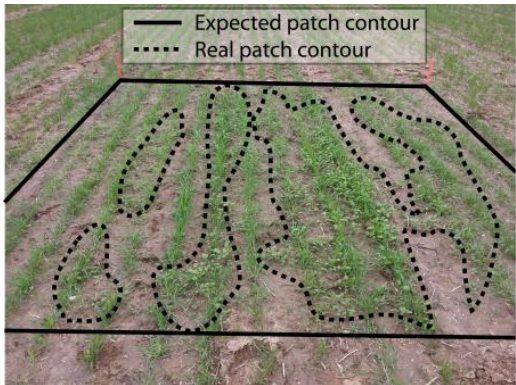

(a)

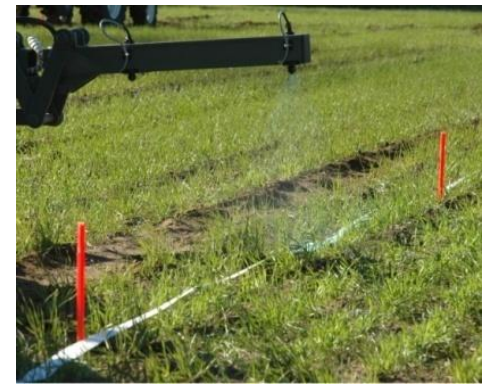

(b)

Fig. 6. a) Real patch vs. expected patch y b) paper strips along the field

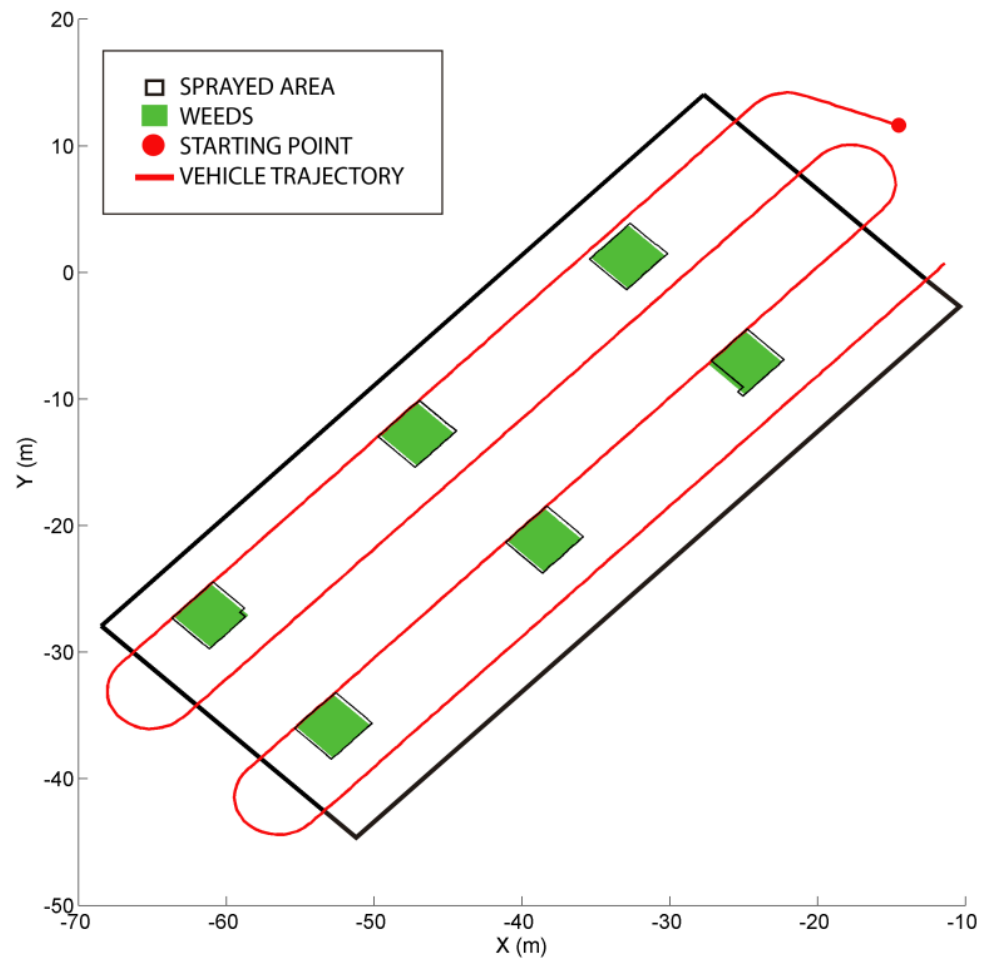

Fig. 7. Ground mission trajectory and sprayed surface 


\section{$5 \quad$ Conclusions and future work}

An approach to properly combine autonomous aerial inspection and autonomous ground intervention missions to address environmental problems was proposed. The approach involves a Mission Manager that allows a single operator to supervise the entire process and manage the workflow required to autonomously complete a mission.

The proposed system was tested by performing a real site-specific weed treatment, in which the scouting mission was used to acquire the data to detect the weed patches positions that allowed the intervention treating only the infested areas and, consequently, reducing the cost of the treatment and the chemical pollution.

All the steps needed to achieve the site-specific weed treatment as well as the management of the workflow required to complete the entire process were entirely automated. Human intervention was only required to launch/land the aerial units (due to the current Spanish regulations) and to input the aerial images into the base station computer, since the camera characteristics did not allow the real-time output of high quality images directly to the computer during the acquisition step.

In future, work image transmission and human intervention will be automated, and the new approach will be tested for other types of applications.

Acknowledgements. This project was funded in part by the $7^{\text {Th }}$ Framework Programme of the European Union under Grant Agreement No. 245986. The authors recognise the following RHEA beneficiaries: CSIC (Spain), CogVis (Austria), FTW (Austria), Cyberbotics (Switzerland), University of Pisa (Italy), University Complutense of Madrid (Spain), Tropical (Greece), AGROSAP (Spain), Polytechnic University of Madrid (Spain), AirRobot (Germany), University of Florence (Italy), IRSTEA (France), CNH (Belgium), Bluebotics (Switzerland) and CM (Italy).

\section{References}

1. Gaudin, S.: MIT builds swimming, oil-eating robots. Computerworld (26 August 2010) http://www.computerworld.com/article/2514966/emerging-technology/mit-buildsswimming--oil-eating-robots.html

2. Casbeer, D. W., Beard, R. W., McLain, T. W., Li, S. M., Mehra, R. K.: Forest fire monitoring with multiple small UAVs. In: American Control Conference 2005 (2005)

3. Nathan, M., Shaojie, S., Kartik, M., Yash, M., Vijay, K., Keiji, N., Yoshito, O., Seiga, K., Kazuki, O., Kazuya, Y., Kazunori, O., Eijiro, T., Satoshi, T.: Collaborative mapping of an earthquake-damaged building via ground and aerial robots. Journal of Field Robotics, 29(5), 832-841 (2012)

4. Marshall, E. J. P: Field-scale estimates of grass weed populations in arable land. Weed Research, 28(3), 191-198. (1988).

5. Johnson, G. A., Mortensen, D. A., \& Martin, A. R.: A simulation of herbicide use based on weed spatial distribution. Weed Research, 35(3), 197-205. (1995).

6. ECPA. Report Annual Review 2007, (2008) http://www.ecpa.be/files/ecpa/documentslive/22/18192_ECPA\%202008\%20Annual\%20re port.pdf 
7. Pimentel, D., Acquay, H., Biltonen, M., Rice, P., Silva, M., Nelson, J., Lipner, V., Giordano, S., Horowitz, A., D'Amore, M.: Environmental and economic costs of pesticide use. BioScience , 42, 750-758 (1992)

8. ECPA. Report Annual Review 2011, (2012). http://www.ecpa.eu/files/attachments/Annual Report_web.pdf

9. Miller G.T.: Sustaining the Earth, 6th edition. Thompson Learning, Inc. Pacific Grove, California. Chapter 9, Pages 211-216 (2004)

10. Pierce, F. J., Nowak, P.: Aspects of Precision Agriculture. Advances in agronomy , 67, 185 (1999)

11. Torres-Sánchez, J., López-Granados, F., De Castro, A. I., Peña-Barragán, J. M.: Configuration and specifications of an unmanned aerial vehicle (UAV) for early site specific weed management. PLoS ONE , 8 (3), (2013)

12. Carballido, J., Perez-Ruiz, M., Gliever, C., Agüera, J.: Design, development and lab evaluation of a weed control sprayer to be used in robotic systems. In: First International Conference on Robotic and Associated High-Technologies and Equipment for Agriculture, 1, pp. 23-29. Pisa (2012)

13. Gerhards, R.: Managing weeds with respect to their spatial and temporal heterogeneity. In: 2nd Conference on Precision Crop Protection. (2007)

14. Ruiz, D., Escribano, C., Fernandez-Quintanilla, C.: Assessing the opportunity for sitespecific management of Avena sterilis in winter barley fields in Spain. Weed Research 46, 379-387 (2006)

15. RHEA project Website, http://www.rhea-project.eu

16. AirRobot Website, http://www.airrobot.de

17. Boomer 3050 Tractor Website, http://agriculture1.newholland.com/nar/enus/equipment/products/tractors-telehandlers/boomer-3000-series/models

18. Emmi, L., Gonzalez-de-Soto, M., Pajares, G., Gonzalez-de-Santos, P.: Integrating Sensory/Actuation Systems in Agricultural Vehicles. Sensors, 14, 4014-4049 (2014)

19. Rabatel, G., Labbé, S.: A fully automatized processing chain for high-resolution multispectral image acquisition of crop parcels by UAV. Precision agriculture, 15(1), 135$141(2015)$

20. RHEA demo, http://www.rhea-project.eu/img/Videos/DEMO_short_version_01.mp4 